\title{
A SEARCH FOR BETA CEPHEI STARS IN LMC AND SMC *
}

\author{
H. KJELDSEN and D. BAADE \\ European Southern Observatory \\ Karl-Schwarzschild-Str. 2, D-85748 Garching bei München, Germany
}

\section{Testing the $\kappa$ mechanism}

The $\beta$ Cephei instability is believed to be caused by the $\kappa$-mechanism. Model calculations, based on revised metal opacities, show this mechanism to be strongly sensitive to metallicity (e.g., Dziembowski, W. A. \& Pamyatnykh, A. A., 1993: $M N R A S, 262,204)$. It appears that $[\mathrm{Fe} / \mathrm{H}]$ must be larger than the solar value in order to drive the $\beta$ Cephei pulsations. For this reason, the models predict that no $\beta$ Cephei stars should be found in the Magellanic Clouds. Balona (1992: MNRAS, 256, 425; 1992: ASP Conf. Ser., Vol. 30, 155; 1993: MNRAS, 260,795) has tested this prediction by observing three young clusters in the SMC and LMC (NGC 330, NGC 2004 and NGC 2100). He found no $\beta$ Cephei stars down to a magnitude of $V \approx 16$; the threshold for detection of variability on time-scales of a few hours was $10 \mathrm{mmag}$. Most Galactic $\beta$ Cephei stars have amplitudes greater than this, but we might expect $\beta$ Cephei stars in the Magellanic Clouds (if they exist) to have smaller amplitudes, due to the different environment. To test this, we have observed two young clusters NGC 371 (SMC) and NGC 2122 (LMC) with high sensitivity.

\section{Observing technique and data reduction}

The data were collected on $2 \times 6$ nights in November-December, 1992 using the $0.91 \mathrm{~m}$ Dutch telescope at La Silla. We did time-resolved high-precision CCD photometry using a $385 \times 578$ pixel GEC CCD and typical exposure times of $120 \mathrm{sec}$. In total we got 1660 usefull time-series frames. All timeseries observations were done through a Bessel V-filter. We also collected $\mathrm{UBV}, \mathrm{H} \beta$ and uvby-Strömgren photometry for both clusters, in order to place the observed stars in the colour-magnitude diagram. The data were reduced using the MOMF software (Kjeldsen, H. and Frandsen, S., 1992: $P A S P, 104,413)$, which was developed specifically for high-precision differential photometry. We have calculated the photometric precision as a function of relative magnitude for NGC 371 and find that, for most stars, the precision is close to the theoretical limit set by photon, sky and detector noise.

* Based on observations obtained at the European Southern Observatory, La Silla, Chile 


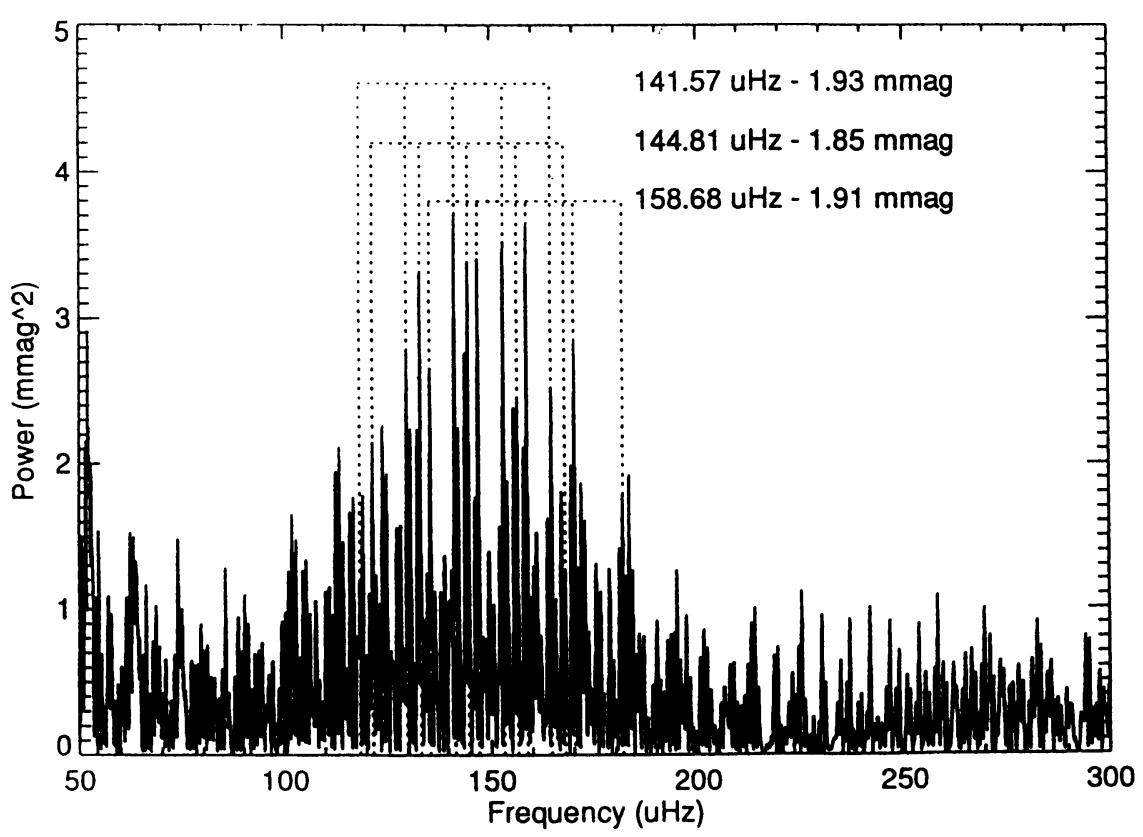

Fig. 1. Amplitude-scaled power spectrum for star \# 565 in NGC 371 (SMC). The spectrum can be explained by three close frequencies convolved with the window function of the observations. The amplitudes are below $2 \mathrm{mmag}$ and frequencies correspond to $12.23,12.51$ and $13.71 \mathrm{c} /$ day (periods: $1.96,1.92$ and 1.75 hours).

\section{Results}

To search for variability, we examined the amplitude spectra for the $\sim 100$ brightest stars in each cluster (down to $V \approx 16$ ). We find no clear evidence for variability on timescales of 1-6 hours above a noise level of 1-4 mmag. The lack of pulsating stars in these metal-poor clusters gives strong support to the $\kappa$-mechanism being the cause of $\beta$ Cephei pulsations.

Two stars in NGC 371 show some indication of pulsations at the level of a few mmag. The signals are close to our sensitivity limit and may simply be peaks in the noise distribution. However, both stars (\#565 and \#642) are located in the $\beta$ Cephei instability strip in the CM-diagram $\left(-5 \lesssim M_{V} \lesssim-4\right)$. If real, the pulsations have amplitudes of 2-3 mmag and periods close to two hours (see Figure 1). We note that Galactic $\beta$ Cephei stars, in comparison, have longer periods (3-6 hours).

Acknowledgements: We thank Tim Bedding and Søren Frandsen for stimulating discussions and help during this project.

(The full results of this project will be submitted to Astronomy \& Astrophysics when the analysis has been completed.) 